\title{
Trigeminal Amyloidoma: Case Report and Review of the Literature
}

\author{
Oren N. Gottfried, M.D., ${ }^{1}$ Steven Chin, M.D., ${ }^{2}$ H. Christian Davidson, M.D., ${ }^{3}$ \\ and WilliamT. Couldwell, M.D., Ph.D.'
}

The authors present a case of amyloid infiltration involving the trigeminal nerve that mimicked a malignant cavernous sinus tumor with perineural tumor infiltration. A 64-year-old man presented with trigeminal nerve numbness. Imaging revealed a plaque-like enhancing lesion along the right lateral cavernous sinus extending anteriorly into Meckel's cave and involving the proximal V2 and V3 branches of the trigeminal nerve. The patient underwent an extradural frontotemporal craniotomy with middle fossa exposure of the cavernous sinus to diagnose and treat the presumed malignant cavernous sinus tumor. A reddish mass involving the lateral dural wall of the cavernous sinus was resected. The gasserian ganglion, V2, and V3, the latter of which was biopsied, were enlarged. Permanent histopathological studies showed microscopic eosinophilic, amorphous material, which stained positive for Congo red, and an absence of neoplastic cells. The final diagnosis was amyloidoma. Thus, amyloidomas can involve the trigeminal nerve or ganglia and should be considered in the differential diagnosis of a cavernous sinus lesion mimicking a tumor. Patients may have symptomatic improvement of trigeminal neuropathy with resection of the amyloidoma outside the nerve capsule that is compressing the nerve, while resection of the lesion from within the capsule may result in permanent trigeminal nerve dysfunction.

KEYWORDS: Gasserian ganglion, trigeminal, amyloidoma

Amyloidomas of the nervous system are extremely uncommon and are invariably of the amyloid light-chain lambda type. ${ }^{1}$ Typically, cerebral amyloidomas occur as single or multiple supra- tentorial masses located in the white matter of the brain parenchyma. ${ }^{1}$ Only rarely has amyloid disease been described to involve other nervous tissue, and only 12 cases of amyloid involving the gasserian

\footnotetext{
${ }^{1}$ Department of Neurosurgery, ${ }^{2}$ Department of Pathology, ${ }^{3}$ Department of Radiology, University of Utah, Salt Lake City, Utah.

Address for correspondence and reprint requests: William $\mathrm{T}$. Couldwell, M.D., Ph.D., Department of Neurosurgery, University of Utah School of Medicine, 175 N. Medical Drive East, Salt Lake City, UT 84132 (e-mail: neuropub@hsc.utah.edu).
}

Skull Base 2007;17:317-324. Copyright (C) 2007 by Thieme Medical Publishers, Inc., 333 Seventh Avenue, New York, NY 10001, USA. Tel: +1(212) 584-4662.

Received: January 25, 2006. Accepted after revision: July 11, 2006. Published online: September 7, 2007.

DOI 10.1055/s-2007-986430. ISSN 1531-5010. 
ganglion or trigeminal nerve have been reported. ${ }^{2-11}$ These patients may present with a pattern of trigeminal amyloidosis, starting with trigeminal anesthesia, hypalgesia, or dysesthesia associated with progressive facial anesthesia and, possibly, weakness or atrophy of the muscles of mastication. ${ }^{12}$ When imaging reveals a mass in Meckel's cave, a mass around the gasserian ganglion or trigeminal branches, or a cavernous sinus lesion, it is important to consider amyloidoma, a benign process, in the differential diagnosis to select the appropriate management and surgical strategy. We report a patient with progressive trigeminal nerve sensory loss from an amyloidoma of the trigeminal nerve; this patient had a dural-based mass and an enlarged trigeminal nerve that mimicked a malignant cavernous sinus tumor with perineural tumor infiltration.

\section{CASE REPORT}

A 64-year-old man had a 9-month history of progressive numbness and tingling on the right side of his face without associated pain. He had decreased sensation in the distribution of V2 and V3 of the trigeminal nerve. Magnetic resonance imaging (MRI) showed a plaque-like enhancing lesion along the right cavernous sinus extending anteriorly into Meckel's cave. The mass involved the proximal V2 and V3, extending to the foramina rotundum and ovale, respectively, and to the vidian nerve. These nerves also appeared enlarged (Fig. 1). The preoperative diagnosis of this lesion was malignant cavernous sinus tumor associated with perineural tumor infiltration or, less likely, meningioma or schwannoma.
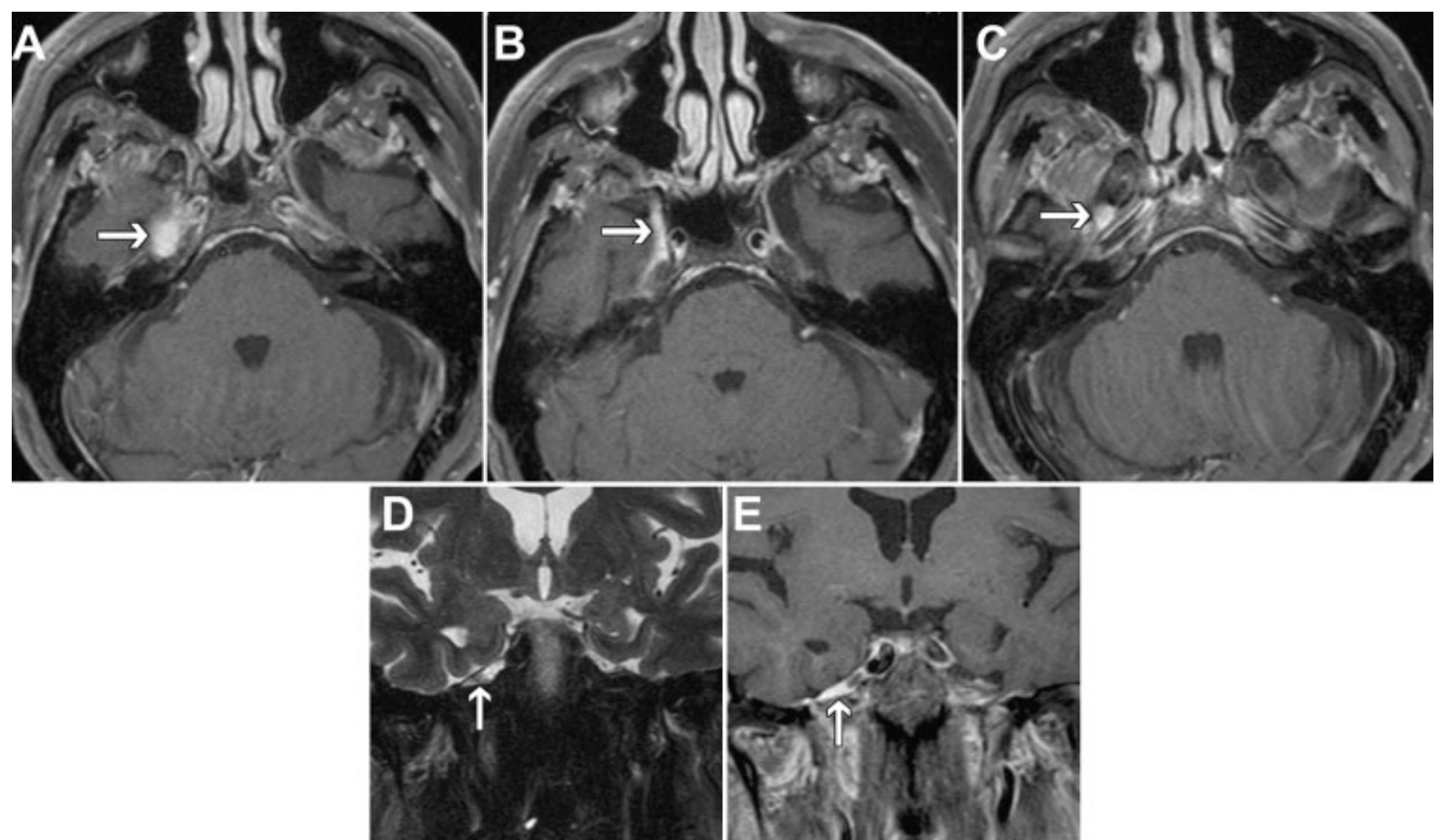

Figure 1 (A-C) Axial T1-weighted magnetic resonance images through the central skull base after contrast administration. (A) Enhancing tissue representing amyloidoma is evident in the lower Meckel's cave region on the right (arrow). (B) Perineural enhancement extends into the foramen rotundum (arrow) and (C) foramen ovale (arrow). Coronal STIR and postcontrast T1-weighted magnetic resonance images. (D) Amyloid deposition appears as a filling defect in the lateral aspect of Meckel's cave on STIR image (arrow). (E) Corresponding enhancement is demonstrated (arrow). 


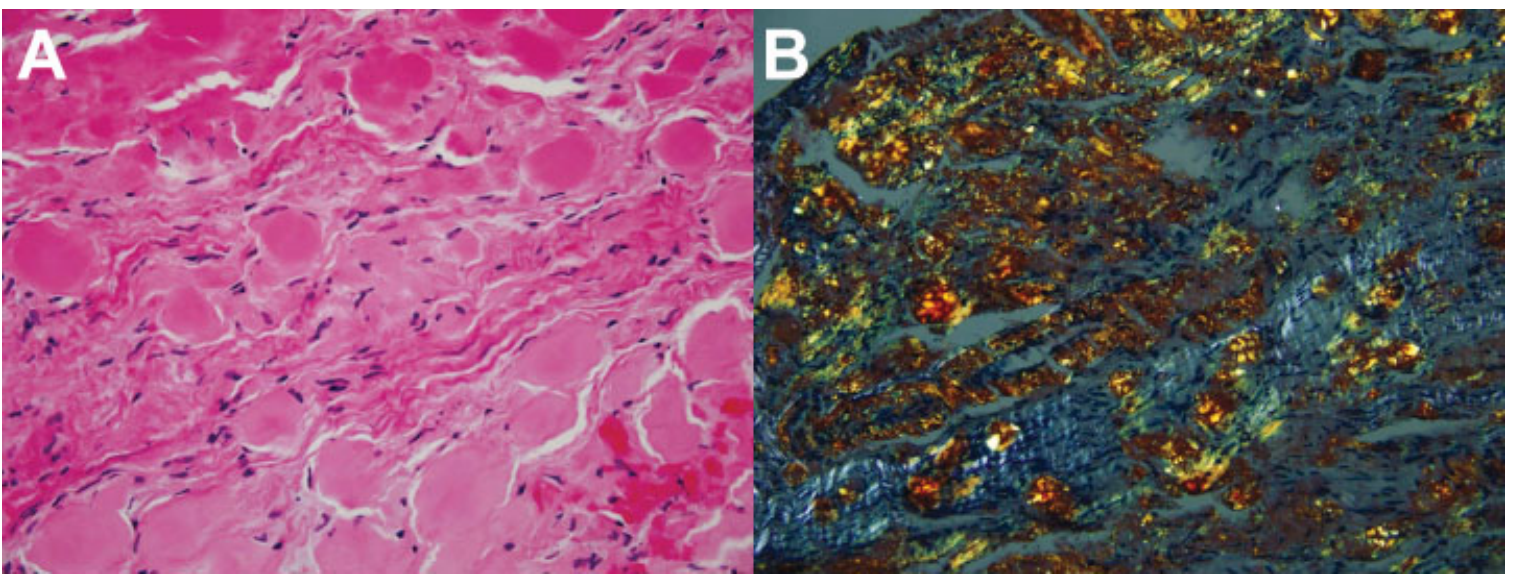

Figure 2 Photomicrograph. (A) Specimen shows deposits of eosinophilic, amorphous material. No significant inflammatory cells or neoplastic cells are present. (Hematoxylin and eosin staining, original magnification, $\times 100$ ). (B) Typical pattern of apple-green birefringence is present in specimen stained with Congo red and viewed with polarized light, $\times 40$.

The results of a chest $\mathrm{x}$-ray, complete blood count, and serum chemistry test were normal. Given the patient's progressive trigeminal symptoms, he was offered surgery for the purpose of diagnosis and possible resection. He underwent a right frontotemporal craniotomy with middle fossa exposure of the cavernous sinus through an extradural exposure. A reddish mass involving the lateral dural wall of the cavernous sinus was noted. The bone around the foramen rotundum and foramen ovale was drilled to afford decompression of the enlarged V2 and V3 branches. After the lateral wall of the cavernous sinus was removed, the trigeminal nerve was inspected. The gasserian ganglion and the V2 and V3 were enlarged and appeared infiltrated, although the fascicular pattern of the nerve was maintained. A biopsy of a fascicle of the V3 at the entrance of the nerve branch through the foramen ovale was obtained.

Permanent histopathological studies showed microscopic eosinophilic, amorphous material, which stained positive for Congo red (Fig. 2) and thioflavine S. Immunohistochemistry studies showed staining for lambda light chains. No neoplastic cells were identified, but reactive meningothelial cells were present. The samples from the dural mass and the fascicle of $\mathrm{V} 3$ were identified to contain extensive amyloid deposition consistent with amyloidoma.
The patient's postoperative course was uneventful. After decompression, sensation in his lower and middle face improved and the improvement has remained stable for more than 1 year of follow-up. Additional studies showed no evidence of systemic amyloidosis. MRI obtained at 1 year after treatment showed some diminished enhancement of the cavernous sinus.

\section{DISCUSSION}

Amyloid is an abnormal insoluble protein. Under certain pathological conditions, it can be deposited in the extracellular space and can involve almost every organ system. ${ }^{13}$ Although amyloid deposition within the brain can take many forms, including cerebral amyloid angiopathy or senile plaques of Alzheimer dementia, tumor-like deposition (amyloidoma) is extremely uncommon. ${ }^{13}$ Amyloidoma is defined as primary solitary amyloidosis where no plasma-cell dyscrasia or abnormal serum proteins are detectable. ${ }^{14}$ The source of amyloid protein that forms a tumor in the central nervous system is unclear. It is thought to derive from components that leak from vessels or to be synthesized at the site of deposition from coexistent plasma cells. However, the origins of intracerebral plasma cells 
observed in some cerebral amyloidomas are not understood. ${ }^{13,15}$

At presentation, the mean age of patients harboring cerebral amyloidomas is 47.8 years, and there is a slight female predominance. ${ }^{13}$ Amyloidomas are located in the brain parenchyma. ${ }^{1}$ Patients have solitary or multiple supratentorial white matter masses that exert little or no mass effect on the surrounding anatomy; they extend medially to the lateral ventricle ependyma and have irregular margins. ${ }^{13}$ Computed tomography (CT) typically shows a mass that varies from hypodense to patchy increased density and contrast enhances. T1weighted MRI of cerebral amyloidoma mostly shows hypointense lesions, but amyloidoma can also appear isointense or hyperintense. On T2weighted MRI, mixed high and low signal intensity can be present, with lesions enhancing to a variable degree. ${ }^{13}$ The clinical course of intracerebral amyloidomas is benign, and these lesions can grow slowly. Occasionally, amyloidoma regrows after resection. ${ }^{13,15}$ However, the natural history of this rare lesion is not fully understood because of the low incidence of the disease.

Amyloidomas have affected other nervous tissue, including spinal roots, ${ }^{16}$ the brachial plexus, ${ }^{17}$ and peripheral nerves. ${ }^{18}$ Rarely, amyloid disease has caused trigeminal neuropathy from its involvement with the gasserian ganglion, and why there is a predisposition for these ganglia is unclear. $^{2-11}$ Overall, there have been 12 reports of patients with trigeminal amyloidomas, beginning with the first case described by Daly et al. in $1957^{4}$ (Table 1). These patients all presented with some form of trigeminal neuropathy, including trigeminal hypalgesia, dysesthesia, or face anesthesia, and rarely with V3 motor dysfunction manifesting as weakness or atrophy of the muscles of mastication. In most cases, the hypalgesia or dysesthesias preceded the onset of facial anesthesia. Over time the distribution of the numbness on the face increased. The duration of symptoms before treatment has ranged from 2 months to as long as 10 years. ${ }^{3}$ In two cases, patients had bilateral trigeminal nerve dysfunction from bilateral amyloidomas. ${ }^{6,8}$
Although all patients presented with trigeminal neuropathy and many with continuous facial pain, the symptoms were distinct from trigeminal neuralgia, which is associated with episodes of intense, stabbing, electric shock-like pain and with specific triggers.

With advances in imaging, it has been possible to improve characterization of these lesions. With increased knowledge of amyloidomas, it may be possible to identify these lesions on imaging before surgery (Table 1 ). In only one study ${ }^{7}$ did the authors describe the density of the amyloidoma on CT, and they found that the tumor was isodense. In contrast to cerebral amyloidomas, these trigeminal variants often appeared isointense on T1weighted MRI and hypointense or of mixed signal intensity (both hypointense and isointense) on T2weighted MRI. The mixed signal intensities of amyloidomas on T2-weighted images have been attributed to the uneven deposition of amyloid protein. $^{7}$ The low signal intensity on T2-weighted imaging related to increased amyloid deposition may suggest the diagnosis of amyloidoma, and this feature may differentiate amyloidoma from other lesions, including schwannomas. ${ }^{10,11}$ In fact, the most common preoperative diagnosis of the 12 reported patients with amyloidomas was schwannoma. Finally, a common characteristic on MRI was uniform enhancement localized to Meckel's cave with enhancement of the ganglion, nerve branches, or both.

Most authors have taken similar approaches to the management of trigeminal amyloidomas (Table 1). Typically, these tumors have been located exclusively within the nerve capsule and have infiltrated in and around the nerve fibers. Many of these patients underwent a craniotomy with incision of the nerve capsule and biopsy of the abnormal tissue without an attempt at further resection. In these cases, follow-up information and imaging were not provided. Thus it is unclear whether the biopsy affected the patients' symptoms or the natural history of the disease.

Several cases have involved resection of the lesion..$^{2-5,7,10}$ Matsumoto and colleagues ${ }^{7}$ resected 


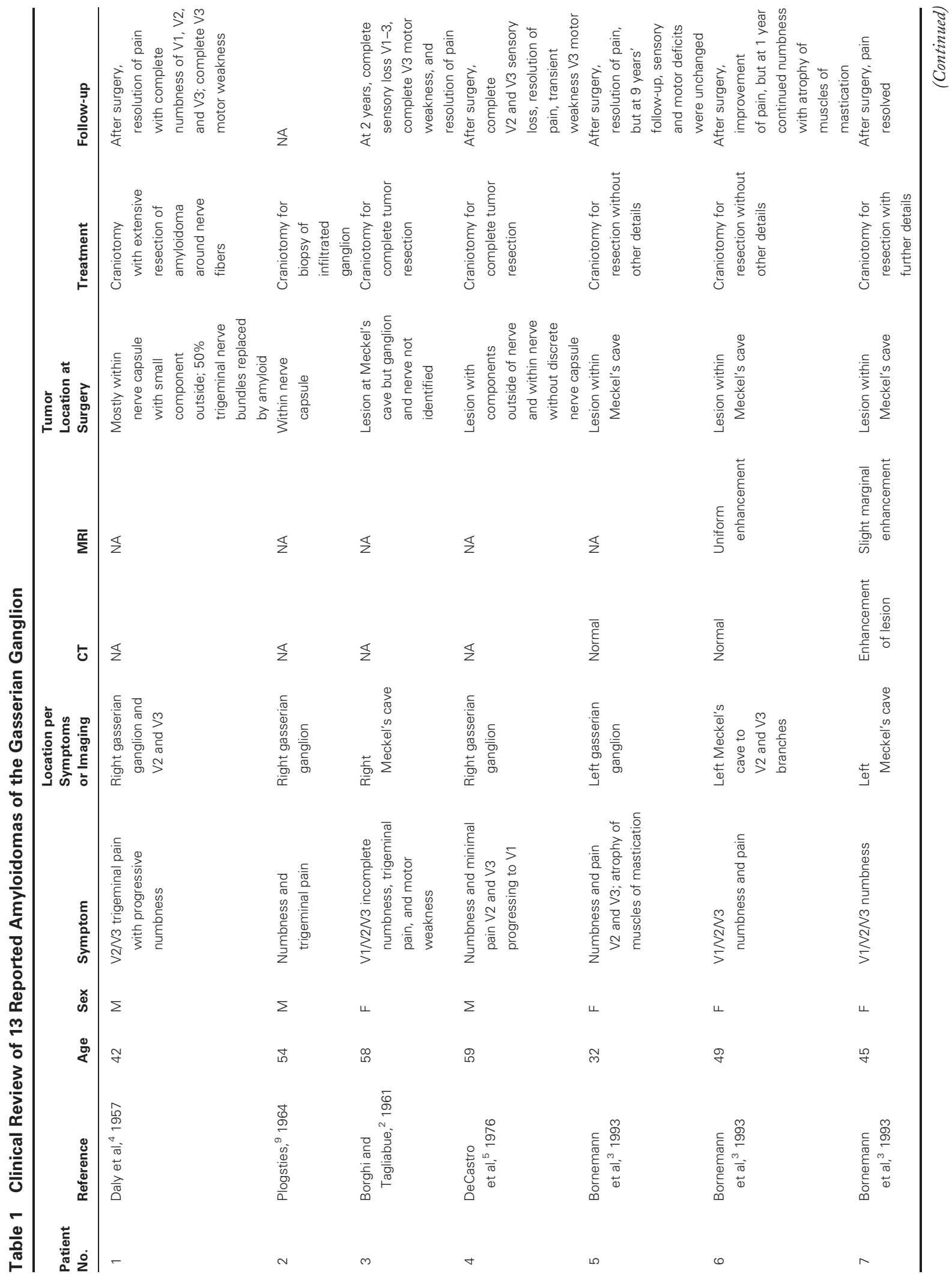




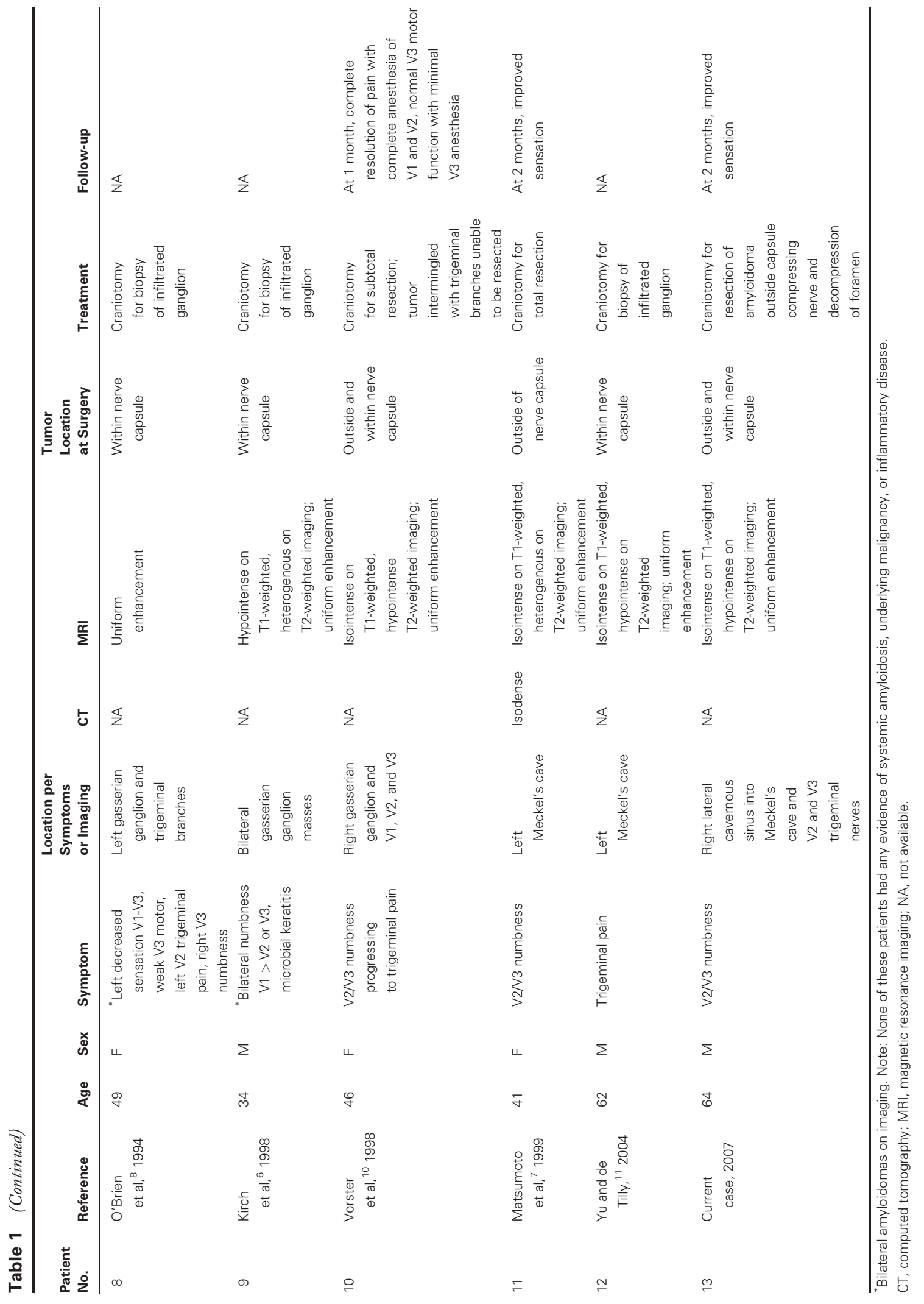


an amyloidoma through an extradural skull base approach and found the tumor located between the lateral wall of the cavernous sinus and the $\mathrm{V} 2$ and V3. The tumor adhered slightly to the trigeminal nerve but was easily removed so that gross total resection was obtained. In a similar surgery, Vorster and coworkers ${ }^{10}$ resected an amyloidoma located medial to the gasserian ganglion, which was clearly distinguishable from it. However, amyloid was intermingled with the V1, V2, and V3 branches. The surgical plane was lost where the lesion was intimately involved with these nerves. Thus, a subtotal resection was performed to spare the function of the nerves. In contrast to the patient described by Matsumoto et al, their patient suffered an increase in numbness from the surgery. In another two reports, ${ }^{2,5}$ it was difficult to differentiate lesion from nerve. Gross total resection was attempted, but both patients had increased sensory loss and weakness after surgery. Finally, the patient described by Daly and coworkers ${ }^{4}$ underwent an extensive piecemeal resection of all of the abnormal tissue intermingling with the nerve fibers within the nerve capsule. After surgery, the patient had complete trigeminal numbness in all distributions and V3 motor weakness. Overall, decompressive surgery with debulking of tumor outside the capsule was associated with improvement or resolution of trigeminal pain and numbness, while extensive resection within the nerve capsule was associated with worse trigeminal dysfunction. ${ }^{2,4,5,10}$

In the current case, the tumor located outside the nerve was resected completely through an extradural approach similar to two of the cases above $^{7,10}$; however, the tumor within the nerve capsule involving the nerve fibers was biopsied but not resected. The V2 and V3 branches were markedly enlarged; thus, we decompressed the foramina rotundum and ovale. The tumor within the trigeminal nerve was not resected to preserve function. This surgical strategy offers immediate symptomatic relief from trigeminal pain and dysesthesia without increasing sensory loss or V3 motor dysfunction. Long-term imaging has not been reported from any of the 12 cases. Therefore, the natural history of the disease and whether the lesion has the capacity to regrow and cause symptoms after subtotal resection are unknown. In one report, ${ }^{3}$ two patients with postoperative deficits were followed for 1 and 9 years, without a clinical recurrence of symptoms.

This case demonstrates that, in addition to meningiomas, schwannomas, malignant cavernous sinus tumors, and inflammatory disorders, amyloidomas should be considered as a possibility in the differential diagnosis of a cavernous sinus lesion or of a lesion arising from the gasserian ganglion or within Meckel's cave.

\section{ACKNOWLEDGMENTS}

The authors acknowledge the superb editorial assistance of Ms. Kristin Kraus.

\section{REFERENCES}

1. Laeng RH, Altermatt HJ, Scheithauer BW, Zimmermann DR. Amyloidomas of the nervous system: a monoclonal B-cell disorder with monotypic amyloid light chain lambda amyloid production. Cancer 1998;82:362-374

2. Borghi G, Tagliabue G. Primary amyloidosis in the gasserian ganglion. Acta Neurol Scand 1961;37:105110

3. Bornemann A, Bohl J, Hey O, et al. Amyloidoma of the gasserian ganglion as a cause of symptomatic neuralgia of the trigeminal nerve: report of three cases. J Neurol 1993; 241:10-14

4. Daly DD, Love JG, Dockerty MB. Amyloid tumor of the gasserian ganglion. J Neurosurg 1957;14:347-352

5. DeCastro S, Sparks JR, Lapey JD, Freidberg SR. Amyloidoma of the gasserian ganglion. Surg Neurol 1976;6:357-359

6. Kirch IE, Beaver HA, Lee AG, Green LK, Tang RA. Microbial keratitis as the manifestation of trigeminal amyloidoma at initial presentation. J Neuroophthalmol 1998;18:192-195

7. Matsumoto T, Tani E, Fukami M, Kaba K, Yokota M, Hoshii Y. Amyloidoma in the gasserian ganglion: case report. Surg Neurol 1999;52:600-603

8. O’Brien TJ, McKelvie PA, Vrodos N. Bilateral trigeminal amyloidoma: an unusual case of trigeminal neuropathy with a review of the literature. Case report. J Neurosurg 1994; 81:780-783

9. Plogsties HR. On amyloid tumors of the gasserian ganglion [in German]. Acta Neurochir (Wien) 1964;12:120-130 
10. Vorster SJ, Lee JH, Ruggieri P. Amyloidoma of the gasserian ganglion. AJNR Am J Neuroradiol 1998;19: $1853-1855$

11. Yu E, de Tilly LN. Amyloidoma of Meckel's cave: a rare cause of trigeminal neuralgia. AJR Am J Roentgenol 2004;182:1605-1606

12. Love S, Bateman DE, Hirschowitz L. Bilateral lambda light chain amyloidomas of the trigeminal ganglia, nerves and roots. Neuropathol Appl Neurobiol 1997;23:512-515

13. Gandhi D, Wee R, Goyal M. CT and MR imaging of intracerebral amyloidoma: case report and review of the literature. AJNR Am J Neuroradiol 2003;24:519-522

14. Bauer WH, Kuzma JF. Solitary "tumors" of atypical amyloidoma (paramyloid). Am J Clin Pathol 1949; 19:1097-1112

15. Eriksson L, Sletten K, Benson L, Westermark P. Tumourlike localized amyloid of the brain is derived from immunoglobulin light chain. Scand J Immunol 1993;37: 623-626

16. McKechnie S, Yang F, Harper CG, et al. Amyloidoma of a spinal root. Neurology 2003;61:834-836

17. Consales A, Roncaroli F, Salvi F, Poppi M. Amyloidoma of the brachial plexus. Surg Neurol 2003;59:418-423; discussion 423

18. Pizov G, Soffer D. Amyloid tumor (amyloidoma) of a peripheral nerve. Arch Pathol Lab Med 1986;110:969-970

\section{Commentary}

$T_{\text {his is an interesting case with a concise and }}$ comprehensive review of amyloidoma involving the trigeminal ganglion. The lesion is rare and almost indistinguishable from other lesions in the parasellar area that the authors have included in the differential diagnosis. Interestingly, no other cranial nerve ganglia were reportedly involved by this pathology. This is another reason that the results of radiation without a tissue diagnosis are unreliable. I commend the authors for bringing this case to our attention and for reviewing the current literature.

Chandranath Sen, M.D. ${ }^{1}$

\section{Commentary}

The authors have described a rare condition involving the lateral wall of the cavernous sinus and trigeminal nerve. Because of the patient's symptoms and the location involved, the lesion could be mistaken for a trigeminal schwannoma or chondrosarcoma. However, the radiological features appeared to be quite distinct-the lesion was hypointense or isointense on T2-weighted MRI, in contrast to these other two tumors. The authors' strategy of extraneural resection and nerve decompression appears to be a good one, especially if the diagnosis can be made by frozen section, and the tumor is distinguished from malignant lesions such as adenoid cystic carcinoma.

Laligam N. Sekhar, M.D., F.A.C.S. ${ }^{1}$
${ }^{1}$ Department of Neurosurgery, St. Lukes-Roosevelt Hospital, New York, New York.

Skull Base 2007;17:324. Copyright (C) 2007 by Thieme Medical Publishers, Inc., 333 Seventh Avenue, New York, NY 10001, USA.
Tel: +1(212) 584-4662.

Published online: September 7, 2007.

DOI 10.1055/s-2007-986431. ISSN 1531-5010.

Tel: +1(212) 584-4662.

Published online: September 7, 2007.

DOI 10.1055/s-2007-986432. ISSN 1531-5010.
Skull Base 2007;17:324. Copyright (C) 2007 by Thieme Medical Publishers, Inc., 333 Seventh Avenue, New York, NY 10001, USA. 